\title{
Spectral moments for the analysis of frequency shift, broadening, and wavevector anisotropy in a turbulent flow
}

\author{
Y. Narita $1,2,3^{*}$
}

\begin{abstract}
Turbulence represents essentially random fluctuations that evolve both spatially and temporally, and appear in various geophysical and space science applications. A spectral moment method is proposed to characterize the turbulence energy spectra in the wavevector and frequency domain in the lowest-order sense. The frequency shift velocity and the random sweeping velocity are obtained from the first-order and second-order moments of the wavenumber-frequency spectra. The maximum extension direction and the elliptic spectral shape are obtained from the second-order moments of the wavevector spectra. The algorithm for the spectral moment computation is presented with synthetic energy spectra and solar wind energy spectra.
\end{abstract}

Keywords: Turbulence energy spectra, Moment calculation, Random sweeping model

\section{Introduction}

Turbulent fluctuations appear commonly in various fluid and gaseous media in geophysical and space science applications, e.g., ocean turbulence, atmospheric turbulence, and plasma turbulence in near-Earth space. The fluctuating fields (flow velocity, density or pressure variation, or electromagnetic field) develop both spatially and temporally. One method to study the turbulent fluctuations is to determine the correlation in the space-time domain (He and Zhang 2006; Zhao and He 2009). The other method, complementary to the space-time correlation, is to determine the energy spectrum in the wavevector and frequency domain as the Fourier transform of the space-time correlation (Kraichnan 1964; Wilczek and Narita 2012).

The wavevector-frequency spectra are accessible by numerical simulations or multi-point measurements using a properly placed sensor array. In the lowest-order picture, the frequencies of the turbulent field are subject to the Doppler shift and broadening caused by the mean

\footnotetext{
*Correspondence: yasuhito.narita@oeaw.ac.at

${ }^{1}$ Space Research Institute, Austrian Academy of Sciences, Schmiedlstraße 6, 8042 Graz, Austria

Full list of author information is available at the end of the article
}

flow and the large-scale variation of the flow (referred to as the random sweeping field), and the wavevector anisotropy (caused, for example, by a large-scale magnetic field in plasma) can be fitted by a set of elliptic energy contour levels.

Here we propose a spectral moment method to characterize the wavevector-frequency spectra of turbulence using a smallest set of parameters: the shift velocity, the sweeping velocity, and the eigenvalues and eigenvectors of the spectral moment tensor. The shift velocity is associated with the phase speed in the observer frame in the turbulent medium. In the case of supersonic or superAlfvénic media such as in the solar wind, the shift velocity should sufficiently be close to the mean flow speed (Doppler shift). The sweeping velocity is associated with the frequency broadening of the energy spectrum around the Doppler shift or the frequency shift when the spectrum is sliced over the frequencies at a given wavenumber. In the limit of hydrodynamic treatment, the sweeping velocity can be of the order of root-mean-square of the large-scale flow variations (Doppler broadening), or can be influenced by changes in the spatial structure. The eigenvalues and the eigenvectors are obtained from the second-order spectral moment tensor in the wavevector domain and can be used to determine the direction of maximum and 
minimum variation directions (or spectral extension directions) and to characterize the elliptic shape of the spectral anisotropy in the wavevector domain. The algorithm for the spectral moments is presented along with examples of the wavevector-frequency spectra using synthetic data and the spacecraft data in the solar wind.

The moment calculations are a common exercise in the gas and plasma kinetic treatment (moments of velocity distribution function) and in the probability theory (moments of probability density function). The spectral moment method has advantages that the analysis needs only the first-order and the second-order moment calculations of the energy spectra and that the method does not require the knowledge on the power-law behavior (spectral index) or even a shape of the spectrum (on reconstruction of the spectra, however, one has to specify the spectral index). The method in the article is developed for the multi-spacecraft data because the method requires the wavevector dependence of the fluctuation energy. Upon using single spacecraft data only, one has to assume Taylor's hypothesis and project the spacecraft frame frequencies onto the wavevector components with respect to the mean magnetic field (Dasso et al. 2005; Matthaeus et al. 1990). The choice of the wavevector spectrum model depends on the applications. The elliptic wavevector spectrum (Carbone et al. 1995; Narita 2014) is implemented in this article. An extension of the reconstruction model to different spectral indices is possible. For the critical balance wavevector spectrum, the large-scale parameter $L$ (Forman et al. 2011) needs to be determined from the observation. For the non-elliptic wavevector anisotropy model (Narita 2015), the shape parameter (the ratio of the coefficients on the parallel and perpendicular wavenumbers) needs to be determined from the observation. In the case of the onedimensional probability distribution $f$ over a domain of $\chi$ (e.g., the frequencies and the wavenumber in this study; other choices such as particle velocities or fluctuation amplitudes are also possible), the zeroth-order moment is estimated as

$$
\chi^{(0)}=\int \mathrm{d} \chi f(\chi) .
$$

The first-order and the second-order moments are estimated, respectively, as

$$
\begin{aligned}
& \chi^{(1)}=\int \mathrm{d} \chi f(\chi) \chi \\
& \chi^{(2)}=\int \mathrm{d} \chi f(\chi)\left(\chi-\chi^{(1)}\right)^{2} .
\end{aligned}
$$

Normalization of the $n$ th-order moments is possible by dividing by the zeroth-order moment.
The goal of the article is to develop an algorithm to determine the shift velocity (the phase speed of the major fluctuation component), the sweeping velocity, and the sense of wavevector anisotropy using the measurement of the energy spectrum in the wavevector-frequency domain. The essence of the analysis is to extract the information on the principal axis (or the maximum and minimum variance directions) using the spectral moments, and to interpret the spectra in the frame of random sweeping model. Using the assumption of Gaussian statistics (which requires the smallest set of free parameters to characterize the energy spectrum in the wavenumber-frequency domain) and a power-law elliptic shape of the wavevector anisotropy, it is possible to reconstruct the energy spectra in the wavevector and frequency domains.

Two different methods are introduced in the article: (1) computation of the spectral moments in the wavenumber-frequency domain and in the wavevector domain and (2) reconstruction of the spectrum using Gaussian statistics or a power-law distribution. The former is an objective, mathematical exercise and the method itself does not require any specific type of fluctuation data as far as the fluctuations belong the same statistical family (so, discontinuities should be avoided in the data). The latter is a subjective, interpretation work and the results depend on the choice of physics model of interest. We use a Gaussian shape of the spectrum both in the wavenumber-frequency domain, and an elliptic power-law spectrum in the wavevector domain. The corresponding physics model is the random sweeping model in the wavenumber-frequency domain (Kraichnan 1964; Narita 2017; Wilczek and Narita 2012) and the random spatial variation model (Gaussian statistics) with an elliptic sense of wavevector anisotropy (Carbone et al. 1995) for power-law spectrum of elliptic wavevector anisotropy.

It is worth noting that turbulence inherits intermittency (Sorriso-Valvo et al. 1999); otherwise, fully Gaussian fluctuations (no intermittency) imply that there is no wave-wave interaction or fluid nonlinearity (like eddy distortion, vortex line entanglement). In the reconstruction method in the wavenumber-frequency domain assuming Gaussian statistics, the analytic derivation was originally proposed for random sweeping field by Kraichnan (1964), for random sweeping field in a mean flow by Wilczek and Narita (2012), and for random sweeping hydromagnetic field in a mean flow by Narita (2017). Intermittency is renormalized into smallscale fluctuating fields and that fields are regarded as frozen-in into the mean flow and the random sweeping flow in an advected fashion without intrinsic turbulent evolution. 


\section{Frequency shift and broadening Random sweeping model}

The lowest-order picture of turbulence frequency spectra (in the Eulerian frame) is a combination of frequency shift and broadening of the energy spectra in the streamwise wavenumber domain (primarily in the mean flow direction). The frequency shift is caused by the mean flow (Doppler shift) or by a linear mode wave such as the Alfvén wave. The frequency shift maps uniquely the streamwise wavenumbers onto the frequencies through the relation $\omega=k_{\mathrm{s}} U_{\mathrm{sft}}$ where $\omega, k_{\mathrm{s}}$, and $U_{\mathrm{sft}}$ denote the Eulerian frequencies, the streamwise wavenumbers, and the frequency shift velocity, respectively. The shift velocity essentially represents the Doppler shift, which is the basis of Taylor's frozen-in flow hypothesis (Taylor 1938). In addition, the frequency broadening occurs whenever (1) the flow has large-scale variations with the zero mean such that the root-mean-square of the frequency deviation (measured from the Doppler-shifted frequency) is linearly proportional to the root-mean-square of the flow velocity variation, so we associate the frequency broadening with the relation $\sqrt{\left\langle\delta \omega^{2}\right\rangle}=k_{\mathrm{s}} U_{\mathrm{swp}}$, by regarding the sweeping velocity $U_{\mathrm{swp}}$ as the root-mean-square of the flow velocity fluctuation $U_{\text {rms }}$. Here the angular bracket denotes the operation of statistical averaging, or (2) wave-wave interactions produce waves with deviating frequencies from the Doppler shift such as linear modes, sideband waves, nonlinear modes (Howes and Nielson 2013). In the spectral moment method, we determine the shift velocity and the broadening velocity from the measurements, and compare with the Doppler shift and broadening.

A convenient representation of the wavenumber-frequency spectra is given by the random sweeping model; that is, the spectrum is a product of the energy spectrum in the wavenumber domain with a Gaussian frequency distribution (Wilczek and Narita 2012). The energy spectrum is constructed or modeled as follows.

$$
E\left(k_{\mathrm{s}}, \omega\right)=\frac{E\left(k_{\mathrm{s}}\right)}{\sqrt{2 \pi k_{\mathrm{s}}^{2} U_{\mathrm{swp}}^{2}}} \exp \left[-\frac{\left(\omega-k_{\mathrm{s}} U_{\mathrm{sft}}\right)^{2}}{2 k_{\mathrm{s}}^{2} U_{\mathrm{swp}}^{2}}\right]
$$

Here $E\left(k_{\mathrm{s}}, \omega\right)$ is the wavenumber-frequency spectral energy, $E\left(k_{\mathrm{s}}\right)$ the wavenumber spectral energy, $U_{\mathrm{sft}}$ the frequency shift velocity, and $U_{\text {swp }}$ the sweeping velocity. The Gaussian frequency distribution reduces to the Dirac delta function, $\delta\left(\omega-k_{\mathrm{s}} U_{\mathrm{sft}}\right)$, in the limit of vanishing sweeping velocity, $U_{\text {swp }} \rightarrow 0$.

An example of the spectrum model is displayed in Fig. 1 for a shift velocity of $U_{\mathrm{sft}}=5 V_{\mathrm{A}}$ and a sweeping velocity of $U_{\text {swp }}=V_{\mathrm{A}} / 2$. in the space plasma context. The wavenumber spectrum is modeled as $E\left(k_{\mathrm{s}}\right)=E_{0} k_{\mathrm{s}}^{-5 / 3}$. The Alfvén speed $V_{\mathrm{A}}$ and the ion gyrofrequency $\Omega_{\mathrm{i}}$ are

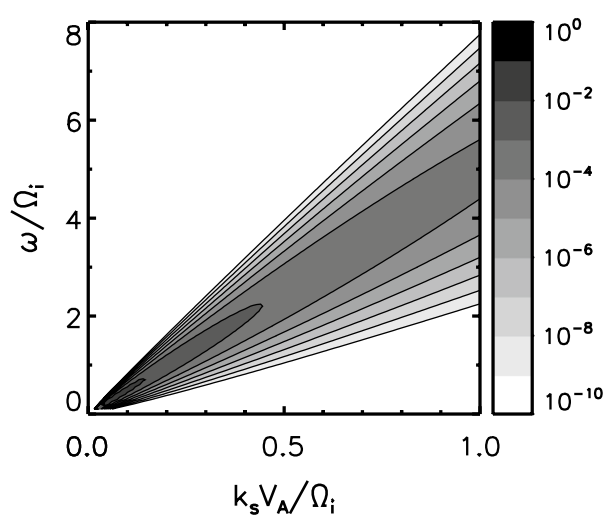

Fig. 1 Energy spectrum in the wavenumber-frequency domain for the random sweeping model. Color bar scale shows levels of the spectral energy density in the wavenumber-frequency domain, so the squared fluctuation amplitude divided by the frequency interval and by the wavenumber interval. The fluctuation amplitudes are normalized to the mean field (e.g., magnetic field), the frequencies to the ion cyclotron frequency, and the wavenumber interval to the ion inertial scale. The color bar scale is normalized here, and reads $E / E_{\omega k}$, where $E_{\omega k}=B_{0}^{2} V_{\mathrm{A}} / \Omega_{\mathrm{i}}^{2}$

used to normalize the velocities and the frequencies, respectively. The wavenumbers are normalized to the ion inertial length, $V_{\mathrm{A}} / \Omega_{\mathrm{i}}$. It is important to note that the assumption of Gaussian statistics is helpful to visualize the spectrum, but is not necessary upon using the spectral moment method. The Gaussian distribution is used only when reproducing or reconstructing the measured spectrum using the first-order and the second-order moments.

\section{Spectral moments}

The spectral moment method extracts the information about the shift velocity $U_{\text {sft }}$ and the sweeping velocity $U_{\mathrm{swp}}$ from the measurement of the spectrum $E\left(k_{\mathrm{s}, \omega}\right)$. The shift velocity is a different quantity from the mean flow velocity, and the shift velocity can be estimated at different wavenumbers. The essence of the method is to cut the spectrum into slices over the frequencies at the selected wavenumbers. Three slices of the model spectrum in Fig. 1 are displayed in Fig. 2 at wavenumbers of $k_{\mathrm{s}} V_{\mathrm{A}} / \Omega_{\mathrm{i}}=\{0.2,0.5,0.8\}$, respectively.

The spectral centers of symmetry (or peaks in the above example) correspond to the peak frequencies associated with the shift velocity (e.g., Doppler-shifted frequency in the hydrodynamic case) and are obtained by estimating the first-order moments. The sliced peak frequencies increase at higher wavenumbers. The mean flow speed is estimated from the first-order moments of the energy spectrum sliced at various wavenumbers. The first-order moment is computed by multiplying the frequency $\omega$ by 


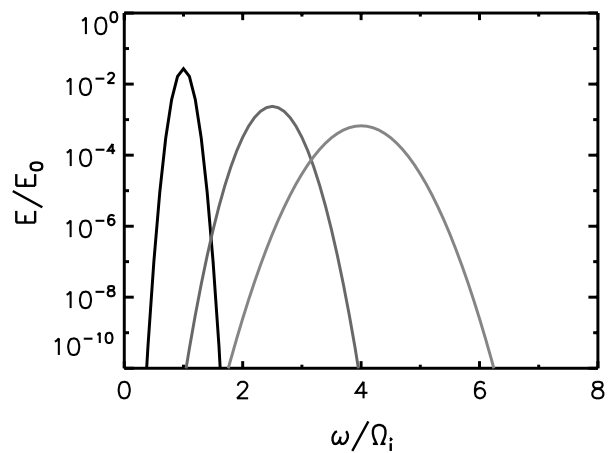

Fig. 2 Slices of the energy spectrum in Fig. 1 at wavenumbers of $k_{\mathrm{S}} V_{\mathrm{A}} / \Omega_{\mathrm{i}}=0.2$ (left curve), $k_{\mathrm{s}} V_{\mathrm{A}} / \Omega_{\mathrm{i}}=0.5$ (middle curve), and $k_{\mathrm{S}} V_{\mathrm{A}} / \Omega_{\mathrm{i}}=0.8$ (right curve)

the spectrum $E\left(k_{\mathrm{s}}, \omega\right)$, and integrating over the frequencies up to the cutoff frequency or over the entire frequency range. The obtained moment represents the peak frequency when normalized to the zeroth-order moment, $\int \mathrm{d} \omega E\left(\omega, k_{\mathrm{s}}\right)$. The shift velocity is obtained by dividing the normalized first-order moment by the slice wavenumber $k_{\mathrm{s}}$ :

$$
U_{\mathrm{sft}}\left(k_{\mathrm{s}}\right)=\frac{1}{k_{\mathrm{s}}} \frac{\int \mathrm{d} \omega E\left(k_{\mathrm{s}}, \omega\right) \omega}{\int \mathrm{d} \omega E\left(k_{\mathrm{s}}, \omega\right)}
$$

The frequency broadening appears as a frequency spread of the sliced spectrum, and increases at higher wavenumbers. The sweeping velocity (e.g., the root-mean-square of flow speed in the hydrodynamic case) is estimated from the second-order moment by multiplying the sliced spectrum by the square of the frequency (measured from the frequency shift as $\left.\omega-k_{\mathrm{s}} U_{\mathrm{sft}}\right)$ integrating over the frequencies, normalizing to the zeroth-order moment, and dividing by the square of the slice wavenumber $k_{\mathrm{s}}^{2}$. The estimator for the squared sweeping velocity is:

$$
U_{\mathrm{swp}}^{2}\left(k_{\mathrm{s}}\right)=\frac{1}{k_{\mathrm{s}}^{2}} \frac{\int \mathrm{d} \omega E\left(k_{\mathrm{s}}, \omega\right)\left(\omega-k_{\mathrm{s}} U_{\mathrm{sft}}\right)^{2}}{\int \mathrm{d} \omega E\left(k_{\mathrm{s}}, \omega\right)}
$$

The advantage of the spectral moment method is that the knowledge on the wavenumber spectra $E\left(k_{\mathrm{s}}\right)$ or slices over the frequencies is not needed because the spectral moments can determined for any physically relevant spectral forms. The information on the frequency shift and broadening is obtained by slicing the energy spectrum at various wavenumbers. Moreover, it is possible to perform a statistics of the shift velocity and the sweeping velocity by regarding the wavenumber slices of the spectrum as an ensemble. Yet, the direction of the streamwise wavenumbers must be known or given by the other means.

\section{Frequency shift and broadening in the solar wind}

The analysis method for the shift velocity $U_{\text {sft }}$ and the sweeping velocity $U_{\text {swp }}$ is tested against the four-point magnetic field data in the solar wind obtained by the Cluster spacecraft (Balogh et al. 2001; Escoubet et al. 2001) for a time interval of March 19, 2005, 03000600 UT. The four Cluster spacecraft formed a nearly regular tetrahedron with a spacecraft separation of about $1000 \mathrm{~km}$. The spacecraft are near the apogee and are almost standing in the solar wind at a distance of about 20 Earth radii ahead of the Earth. Figure 3 displays the magnetic field magnitude measured by the fluxgate magnetometer (Balogh et al. 2001) and the ion bulk velocity and the ion number density measured by the ion electrostatic analyzer (Rème et al. 2001) as time series plots.

The mean magnetic field is nearly dawnward, $(1.0,-6.4,1.4 \mathrm{nT})$ in the GSE (geocentric, solar, ecliptic) coordinate system, with a mean magnitude of about $6.7 \mathrm{nT}$. The mean ion bulk velocity is nearly anti-sunward $(-438,34,51 \mathrm{~km} / \mathrm{s})$ in GSE with a mean magnitude of about $442.2 \mathrm{~km} / \mathrm{s}$, and $(433,0,-90 \mathrm{~km} / \mathrm{s})$ in the mean field-aligned (MFA) coordinate system with the $z$ axis pointing in the direction of the mean magnetic field and the $x-z$ plane spanning the mean magnetic field and the mean ion bulk velocity. The mean ion number density is $7.4 \mathrm{~cm}^{-3}$. The standard deviation of the magnetic field vector fluctuations is $(2.2,2.2,3.3 \mathrm{nT})$ and that of the ion bulk velocity fluctuations is $(15,17,16 \mathrm{~km} / \mathrm{s})$ in the GSE coordinate system. The observation is made in a slow solar wind.

The angle between the mean magnetic field and the mean ion bulk velocity is about $102^{\circ}$. Even though the observation is made upstream of quasi-perpendicular shock (after a crossing at about 0130 UT), there is a moderate, intermittent high-frequency activity at frequencies from 20 to $80 \mathrm{kHz}$ primarily between 0430 and $0500 \mathrm{UT}$,

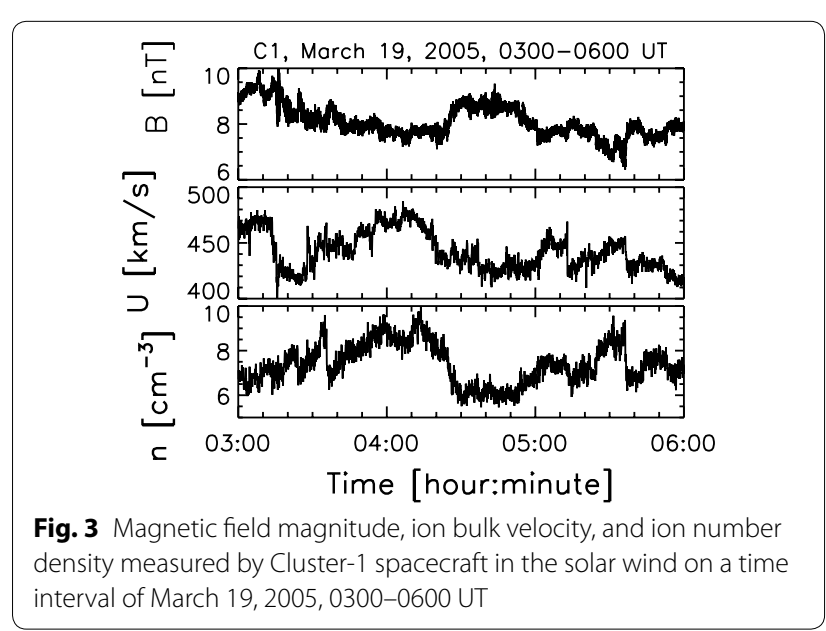


presumably associated with the electron foreshock. We note that the analyzed time interval is moderately mixed by the electron foreshock activity.

The energy spectrum is determined in the streamwise wavenumber-frequency domain in the spacecraft frame (Eulerian frame) from the four-point magnetic field data using the wave telescope (multi-point signal resonator extension or MSR extension), which is a combination of the minimum variance projection with the eigenvaluebased projection (Narita et al. 2011). Figure 4 exhibits a slice of the magnetic energy spectrum as a function of the streamwise wavenumbers $k_{\mathrm{s}}$ and the spacecraft frame frequencies $\omega_{\mathrm{sc}}$ (angular frequencies). The spectrum exhibits an extension with a peak line and a broadening around the peak line. The spectral extension (peak line) is analyzed to determine the shift velocity $U_{\text {sft }}$ and the frequency broadening around the peak line to determine the sweeping velocity $U_{\text {swp. }}$.

The spectral moments are evaluated at various wavenumbers up to the Nyquist wavenumber $\left(3.0 \times 10^{-3} \mathrm{rad} / \mathrm{km}\right)$. Figure 5 displays three examples of the frequency slice of the energy spectrum at wavenumbers of $1.0 \times 10^{-3} \mathrm{rad} / \mathrm{km}$ (top panel), $1.5 \times 10^{-3} \mathrm{rad} / \mathrm{km}$ (middle panel), and $2.0 \times 10^{-3} \mathrm{rad} / \mathrm{km}$ (bottom panel). From the moment calculation, we obtain shift velocities and sweeping velocities $\left(U_{\mathrm{sft}}, U_{\mathrm{swp}}\right)=(396,82 \mathrm{~km} / \mathrm{s})$ at a wavenumber of $1.0 \times 10^{-3} \mathrm{rad} / \mathrm{km}, \quad\left(U_{\mathrm{sft}}, U_{\mathrm{swp}}\right)=(425,85 \mathrm{~km} / \mathrm{s}) \quad$ at a wavenumber of $1.5 \times 10^{-3} \mathrm{rad} / \mathrm{km}$, and $\left(U_{\mathrm{sft}}, U_{\mathrm{swp}}\right)=$ $(401,76 \mathrm{~km} / \mathrm{s})$ at a wavenumber of $2.0 \times 10^{-3} \mathrm{rad} / \mathrm{km}$. The Gaussian frequency distributions are over-plotted using the shift and sweeping velocities in each panel.

The shift velocity and the sweeping velocity are determined as a function of the wavenumbers, and are shown as histograms in Fig. 6. The distributions maximize at a shift velocity of about $400 \mathrm{~km} / \mathrm{s}$ and sweeping velocities between 80 and $100 \mathrm{~km} / \mathrm{s}$. The mean ion bulk speed is $443 \mathrm{~km} / \mathrm{s}$, so the shift velocity obtained from the multipoint magnetometer data is smaller than the flow speed by about $10 \%$. The sweeping velocity is larger than the root-mean-square of the ion bulk speed fluctuation $17 \mathrm{~km} / \mathrm{s}$ and is closer to the Alfvén speed $54 \mathrm{~km} / \mathrm{s}$ estimated from the mean magnetic field and the mean ion density. The sound speed $c_{\mathrm{s}}=\sqrt{\gamma p_{\mathrm{th}} / \rho}=\sqrt{\gamma k_{\mathrm{B}} T / m}$ is estimated as $56 \mathrm{~km} / \mathrm{s}$, where $\gamma=5 / 3$ is the polytropic index, $p_{\text {th }}=n k_{\mathrm{B}} T$ the thermal pressure, $\rho=m n$ the ion mass density, $m$ the proton mass, $n$ the ion number density, $k_{\mathrm{B}}$ the Boltzmann constant, and $T=0.23 \mathrm{MK}$ the ion temperature. Magnetosonic speed is $\sqrt{V_{\mathrm{A}}^{2}+c_{\mathrm{s}}^{2}}=78$ $\mathrm{km} / \mathrm{s}$.

Uncertainties in the shift velocity and the sweeping velocity are estimated as follows.

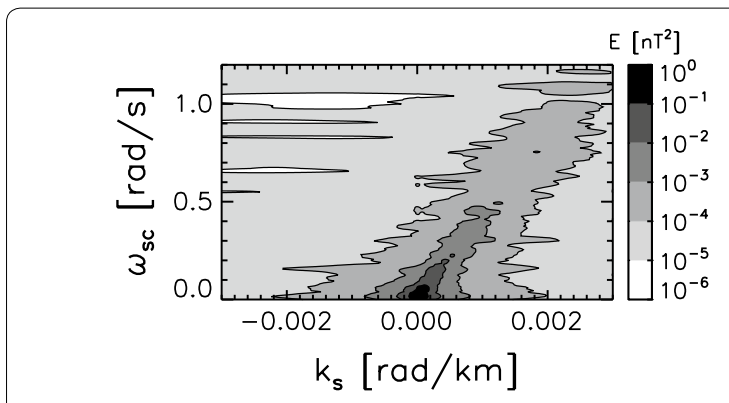

Fig. 4 Streamwise wavenumber-frequency spectrum for magnetic field fluctuations in the solar wind
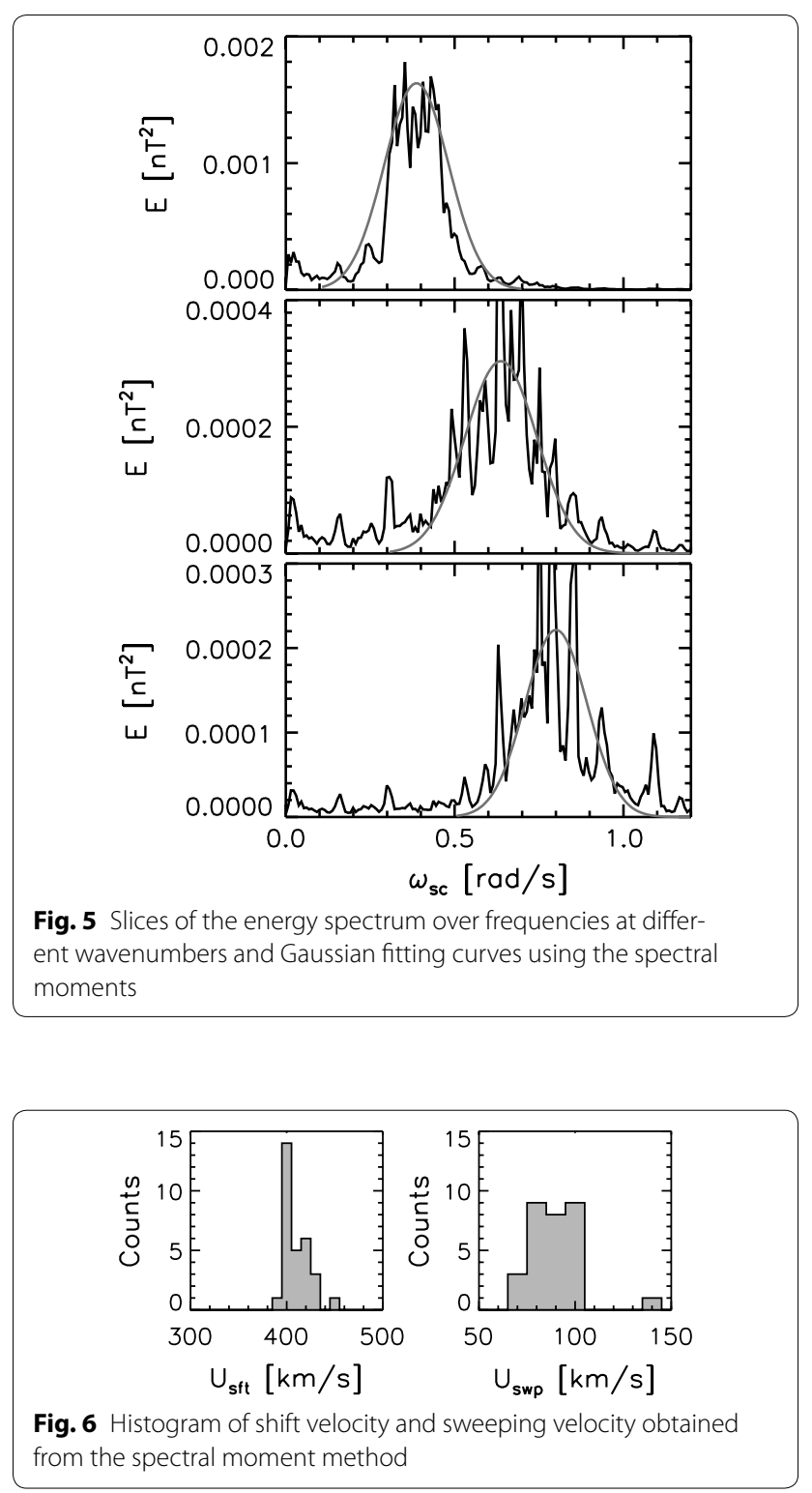
1. The uncertainty in the shift velocity estimate is taken from the standard error by using the central limit theorem. That is, the true shift velocity $U_{\mathrm{sft}(0)}$ is within a interval of

$$
U_{\mathrm{sft}}-\frac{2 U_{\mathrm{swp}}}{\sqrt{n}}<U_{\mathrm{sft}(0)}<U_{\mathrm{sft}}+\frac{2 U_{\mathrm{swp}}}{\sqrt{n}}
$$

at a probability of $95 \%$. Here $n$ stands for the degree of freedom. We use the number of time subintervals in the analysis $(n=24)$. Note that the sweeping velocity is proportional to the standard deviation of the frequency-sliced spectrum with the difference in the factor of the streamwise number $k_{\mathrm{s}}$. The standard error of the shift velocity is

$$
\Delta U_{\mathrm{sft}}=\frac{U_{\mathrm{swp}}}{\sqrt{n}},
$$

and it is estimated as $\Delta U_{\mathrm{sft}}=17 \mathrm{~km} / \mathrm{s}$ at $k_{\mathrm{s}}=1.0 \times 10^{-3} \mathrm{rad} / \mathrm{km}, \quad \Delta U_{\mathrm{sft}}=17 \mathrm{~km} / \mathrm{s} \quad$ at $k_{\mathrm{s}}=1.5 \times 10^{-3} \mathrm{rad} / \mathrm{km}$, and $\Delta U_{\mathrm{sft}}=15 \mathrm{~km} / \mathrm{s}$ at $k_{\mathrm{s}}=2.0 \times 10^{-3} \mathrm{rad} / \mathrm{km}$, respectively.

2. The uncertainty in the sweeping velocity is estimated using the chi-squared distribution with $n-1$ degrees of freedom, $\chi_{n-1}^{2}$. The chi-squared distribution has a variance of $2(n-1)$ (Lehmann and Casella 1998). The standard error of the squared sweeping velocity is

$$
\Delta U_{\mathrm{swp}}=U_{\mathrm{swp}} \sqrt{\frac{1}{2(n-1)}}
$$

for a sufficiently large value of $n$ (typically $n>10$ ). See, for example, Eq. 19 in Ref. Harding et al. (2014). From Eq. (9), we obtain the standard error of the sweeping velocity estimate as $\Delta U_{\text {swp }}=12 \mathrm{~km} / \mathrm{s}$ at $k_{\mathrm{s}}=1.0 \times 10^{-3} \mathrm{rad} / \mathrm{km}, \quad \Delta U_{\mathrm{swp}}=13 \mathrm{~km} / \mathrm{s} \quad$ at $k_{\mathrm{s}}=1.5 \times 10^{-3} \mathrm{rad} / \mathrm{km}$, and $\Delta U_{\mathrm{swp}}=11 \mathrm{~km} / \mathrm{s}$ at $k_{\mathrm{s}}=2.0 \times 10^{-3} \mathrm{rad} / \mathrm{km}$ using $n=24$.

The facts that the shift velocity has an offset from the flow speed by about $10 \%$ and that the sweeping velocity is of the order of the Alfvén speed are interpreted as follows. First, Alfvén waves exist in the solar wind, propagating both sunward and anti-sunward in the plasma co-moving frame and contributing to the frequency broadening. Second, there is more energy in the sunward-propagating Alfvén waves such that the Doppler shift becomes weaker (or frequency becomes lower) than the simple flow advection case without waves. Table 1 displays the velocity measurements (shift velocity and sweeping velocity) from the spectral analysis and compares with the theoretically expected values (ion bulk speed, Alfvén speed, and sound speed).
Table 1 Measured shift velocity and sweeping velocity in comparison to the theoretical estimate for the ion bulk

\begin{tabular}{|c|c|c|c|}
\hline $\begin{array}{l}\text { Streamwise } \\
\text { wavenumber } k_{\mathrm{s}}\end{array}$ & $\begin{array}{l}\text { Shift velocity } \\
\text { measurement }\end{array}$ & \multicolumn{2}{|c|}{$\begin{array}{l}\text { lon bulk speed with Alfvén } \\
\text { speed } U_{\text {flow }} \pm V_{\mathrm{A}}(\mathrm{km} / \mathrm{s})\end{array}$} \\
\hline $1.0 \times 10^{-3}$ & $396 \pm 17$ & \multirow{3}{*}{$443 \pm 54$} & \multirow[b]{4}{*}{$\begin{array}{l}\text { Sound } \\
\text { speed } c_{\mathrm{s}} \\
(\mathrm{km} / \mathrm{s})\end{array}$} \\
\hline $1.5 \times 10^{-3}$ & $423 \pm 17$ & & \\
\hline $2.0 \times 10^{-3}$ & $401 \pm 15$ & & \\
\hline $\begin{array}{l}\text { Streamwise } \\
\text { wavenumber } k_{\mathrm{s}} \\
(\mathrm{rad} / \mathrm{km})\end{array}$ & $\begin{array}{l}\text { Sweeping velocity } \\
\text { measurement } \\
U_{\text {swp }} \pm \Delta U_{\text {swp }}(\mathrm{km} / \mathrm{s})\end{array}$ & $\begin{array}{l}\text { Alfvén } \\
\text { speed } V_{A} \\
(\mathrm{~km} / \mathrm{s})\end{array}$ & \\
\hline $1.0 \times 10^{-3}$ & $82 \pm 12$ & \multirow{3}{*}{54} & \multirow{3}{*}{56} \\
\hline $1.5 \times 10^{-3}$ & $85 \pm 13$ & & \\
\hline $2.0 \times 10^{-3}$ & $76 \pm 11$ & & \\
\hline
\end{tabular}
speed, Alfvén speed, and sound speed using the mean values of plasma and magnetic field data

The error in the spectral velocity measurements is not significant, being between 10 and $20 \mathrm{~km} / \mathrm{s}$. The measured shift velocity is closest to the ion bulk speed subtracted by the Alfvén speed. The analyzed time interval is not so representative of the solar wind property, as one expects Alfvén waves propagating radially away from the Sun, not toward the Sun. The measured sweeping velocity is of the order of Alfvén speed $V_{\mathrm{A}}$ and the sound speed $c_{\mathrm{S}}$, and is closest to the magnetosonic speed $\sqrt{V_{\mathrm{A}}^{2}+c_{\mathrm{s}}^{2}}$.

\section{Wavevector anisotropy}

\section{Spectral moment matrix}

Turbulent fluctuations can be anisotropic in the wavevector domain imposed by a boundary effect or a large-scale magnetic field. If the Eulerian frequencies are limited to the positive domain and the turbulent fluctuations are measured in a flow, the spectral extension reasonably reflects the direction of the mean flow (hereafter the maximum extension direction).

The wavevector anisotropy is characterized by estimating the moment tensor $\boldsymbol{\kappa}$ from the energy spectrum $E(\vec{k})$. The moment tensor is a $2 \times 2$ or a $3 \times 3$ matrix, depending on the dimension of the wavevectors. The moment tensor is obtained by evaluating the second-order moments of the spectrum for two different components of the wavevectors and by normalizing to the zerothorder moment:

$$
\kappa=\frac{\int \vec{k} \vec{k} E(\vec{k}) \mathrm{d} \vec{k}}{\int E(\vec{k}) \mathrm{d} \vec{k}}
$$

Hereafter we concentrate on the wavevector anisotropy in two dimensions for simplicity. The moment 
tensor $\boldsymbol{\kappa}$ is composed of real numbers, and is symmetric with respect to the matrix transpose. The tensor can be diagonalized into a set of eigenvalues $\lambda_{1}$ and $\lambda_{2}$ using the associated eigenvectors, $\vec{e}_{1}$ and $\vec{e}_{2}$, respectively. Since the eigenvectors are mutually orthogonal, the second-order moment representation of the wavevector anisotropy is equivalent to a fitting of the wavevector spectrum by an ellipse characterized by the two eigenvalues (and the associated eigenvectors) as the semi-major and semiminor axes in the wavevector domain. The ratio of the eigenvalues $\lambda_{2} / \lambda_{1}$ serves as a measure of the wavevector anisotropy. The eigenvalues represent typical wavenumbers in the maximal and the minimal spectral extension directions pointed by the eigenvectors.

The ratio of the second-order moments $\lambda_{2} / \lambda_{1}$ can be simplified when the integration is replaced by a sum over the wavevectors (Saito et al. 2008; Shebalin et al. 1983):

$$
\frac{\lambda_{2}}{\lambda_{1}} \simeq \frac{\sum_{\vec{k}}\left(k_{2}\right)^{2} E\left(k_{1}, k_{2}\right)}{\sum_{\vec{k}}\left(k_{1}\right)^{2} E\left(k_{1}, k_{2}\right)},
$$

where $k_{1}$ and $k_{2}$ are the wavenumbers in the maximum and minimum extension directions identified as the eigenvectors of the moment tensor, respectively.

\section{Anisotropy analysis}

The moment tensor method is tested against the synthetic data. The energy spectrum is modeled using an elliptic power law as:

$$
E\left(k_{x}, k_{y}\right) \propto\left(c_{x}^{2} k_{x}^{2}+c_{y}^{2} k_{y}^{2}\right)^{-\alpha}
$$

Figure 7 left panel shows an example of the spectrum model for a ratio of $c_{y} / c_{x}=1 / 2$ and a slope of $\alpha=2$, and the maximum extension direction is tilted from the $x$ axis by $30^{\circ}$.

The eigenvalues and the eigenvectors are determined for the spectrum model. We obtain eigenvalues of $\lambda_{1} \simeq 0.084$ and $\lambda_{2} \simeq 0.040$ and a tilt angle of about $33^{\circ}$ between the maximum extension direction (the eigenvector for the largest eigenvalue) and the $x$ axis (reference axis). The wavevector anisotropy for the example case is thus characterized by an elliptic ratio of $\epsilon=\frac{\lambda_{2}}{\lambda_{1}} \simeq 2.1$ and a tilt angle of $\theta \simeq 33^{\circ}$. Both the eigenvalue ratio and the tilt angle reasonably reproduce the true anisotropy properties $\left(c_{y} / c_{x}=1 / 2\right.$ and $\left.\theta=30^{\circ}\right)$ though the results are not exactly the same as the true values. The systematic error is estimated in more detail below.

Using the eigenvalues and the eigenvectors, the wavevector spectrum can be reconstructed by using a model spectrum. We use a Gaussian function in the synthetic data analysis for the sake of contrast here, and use a power-law function in the spacecraft data analysis

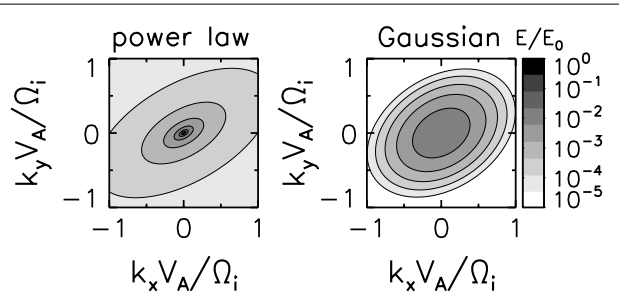

Fig. 7 Elliptic power-law spectrum (left panel) and a Gaussian fit using the moment tensor method (right panel). Color bar scale shows levels of the spectral energy density in the two-dimensional wavevector domain, so the squared fluctuation amplitude divided by the square of wavenumber interval. The fluctuation amplitudes are normalized to the mean field (e.g., magnetic field) and the wavenumber interval to the ion inertial scale. The color bar scale is normalized here, and reads $E / E_{k k}$, where $E_{k k}=B_{0}^{2} V_{A}^{2} / \Omega_{i}^{2}$

(shown below). The spectrum is centered at the origin of the wavevector domain and characterized by the two eigenvalues:

$$
E\left(k_{1}, k_{2}\right)=\frac{1}{2 \pi \sqrt{\lambda_{1} \lambda_{2}}} \exp \left[-\left(\frac{k_{1}^{2}}{2 \lambda_{1}}+\frac{k_{2}^{2}}{2 \lambda_{2}}\right)\right]
$$

where the wavevector coordinates are transformed into the $x-y$ reference system as:

$$
\begin{aligned}
& k_{x}=k_{1} \cos \theta+k_{2} \sin \theta \\
& k_{y}=-k_{1} \sin \theta+k_{2} \cos \theta .
\end{aligned}
$$

The Gaussian fit of the wavevector anisotropy is displayed in Fig. 7 right panel.

Three different studies are performed using the synthetic spectrum.

1. Maximum extension directions are determined for different values of spectral slopes $(\alpha=\{1,2,3\})$ at a fixed value of the elliptic shape $\epsilon_{\text {true }}=c_{y} / c_{x}=1 / 2$. Figure 8 top panel displays the observed (or measured) tilt angle $\theta_{\text {obs }}$ against the true tilt angle $\theta_{\text {true }}$. The tilt angle is within an error of about $6^{\circ}$ (Fig. 9).

2. Maximum extension directions are determined for different values of elliptic shape $\left(\epsilon_{\text {true }}=\{0.05,0.1,0.5\}\right)$ at a fixed spectral slope $\alpha=2$. Again, the observed tilt angle is almost linearly proportional to the true tile angle within an error of about $3^{\circ}$ (Figs. 8 middle panel, 9).

3. Elliptic shapes (the ratio of eigenvalues) are evaluated for different values of spectral slopes $(\alpha=\{1,2,3\})$ at a fixed tilt angle $\left(\theta=0^{\circ}\right)$. The observed eigenvalue ratios $\epsilon_{\text {true }}$ monotonously increase with the true ratios $\epsilon_{\text {true. }}$ The increase rate is larger at smaller values of the elliptic ratio (Fig. 8 bottom panel). 

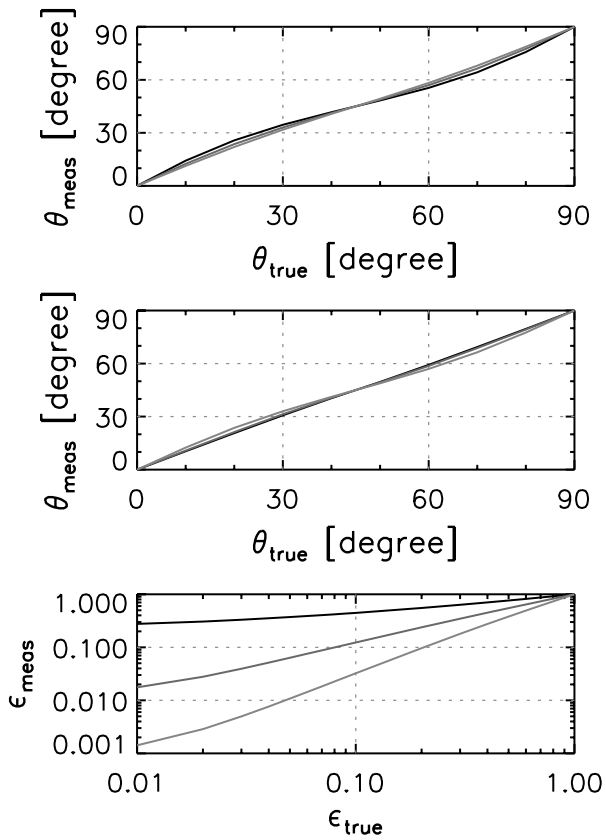

Fig. 8 Observed tilt angle of wavevector anisotropy as a function of the true tilt angle at different values of spectral slopes (top panel) $\alpha=1$ (black), $\alpha=2$ (gray), and $\alpha=3$ (light gray). The same angle relation at different elliptic ratios $\epsilon_{\text {true }}=0.05$ (black), $\epsilon_{\text {true }}=0.1$ (gray), and $\epsilon_{\text {true }}=0.5$ (light gray). The observed elliptic ratio as a function of the true ratio at different values of spectral slopes (bottom panel) $\alpha=1$ (black), $\alpha=2$ (gray), and $\alpha=3$ (light gray)
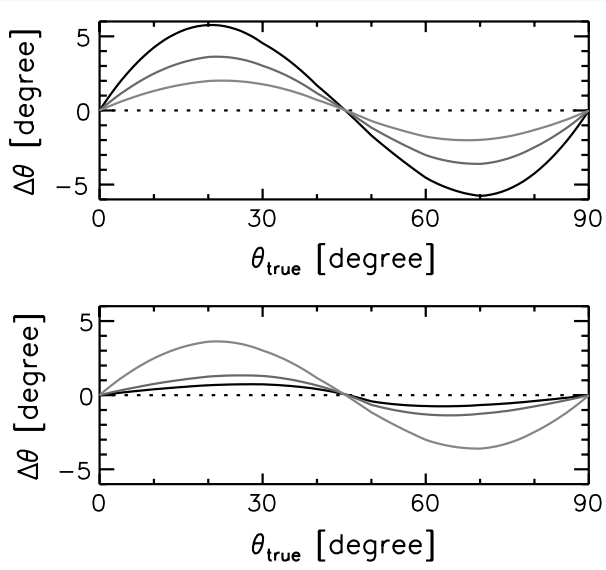

Fig. 9 Tilt angle deviations of the measured principal axis from the true anisotropy direction (spectral extension direction) for Fig. 8 top and middle panels. Top panel shows the angle mismatch for different values of spectral slopes: $\alpha=1$ (black), $\alpha=2$ (gray), and $\alpha=3$ (light gray). Bottom panel shows the angle mismatch for different elliptic ratios $\epsilon_{\text {true }}=0.05$ (black), $\epsilon_{\text {true }}=0.1$ (gray), and $\epsilon_{\text {true }}=0.5$ (light gray)

\section{Wavevector anisotropy in the solar wind}

The spectral moment method is applied to the magnetic energy spectrum in the wavevector domain using the same solar wind data as that shown in Fig. 3. Figure 10 left panel displays the energy spectrum (integrated over the frequencies) in the parallel and perpendicular wavevector components to the mean magnetic field.

We construct the spectral moment matrix $\kappa_{i j}$ and determine the eigenvalues $\lambda_{1}$ (maximum variance) and $\lambda_{2}$ (minimum variance) and the associated eigenvectors $\vec{e}_{1}$ and $\vec{e}_{2}$. The eigenvalue ratio $\lambda_{2} / \lambda_{2}$ is about 0.32 . The maximum and minimum variance directions in the wavevector spectrum show an offset angle from the mean magnetic field by $76.9^{\circ}$.

The energy spectrum is reconstructed using a powerlaw elliptic spectrum (Carbone et al. 1995) with a onedimensional spectral index of $-5 / 3$,

$$
E\left(k_{1}, k_{2}\right) \propto\left[\frac{\lambda_{1}}{\lambda_{2}}\left(k_{1}\right)^{2}+\left(k_{2}\right)^{2}\right]^{-5 / 6}
$$

in the domain spanning the wavevector components in maximum and minimum variance directions, $k_{1}$ and $k_{2}$, respectively. The reconstructed or fitted spectrum is displayed in the right panel of Fig. 10. In this way, an overall structure or shape of the spectrum in the wavevector domain can be reproduced using only a small set of parameters.

The spectral moment method extracts the information on the principal axis and its ratio to the minor axis automatically from the second-order moment calculations. It is true that the method can detect only one spectral extension direction, and if double-anisotropy exists, the spectral moment method cannot properly reproduce the secondary anisotropy direction; The secondary anisotropy is lost by being integrated as an artificial broadening in the direction to the secondary spectral extension. Studying anisotropy cases with more power in the parallel directions (Bruno and Telloni 2015; He et al. 2012) would be an important application to further test the validity of the spectral moment method.

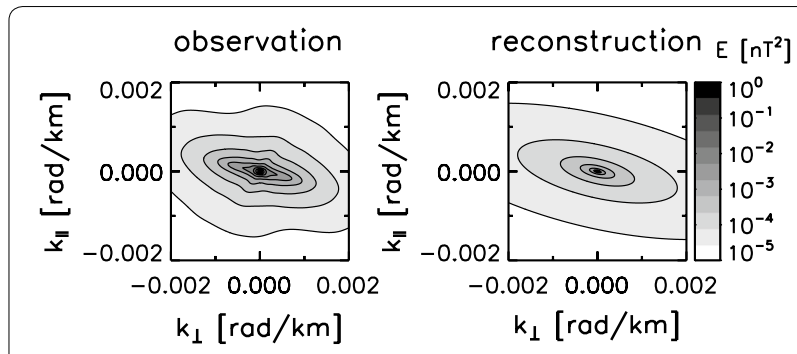

Fig. 10 Cluster observation of wavevector spectrum in the solar wind (left panel) and reconstruction using the spectral moments (eigenvalue ratio of 0.32 and eigenvectors of the spectral moment tensor) and the elliptic power-law model (right panel). The flow direction is normal to the panel 


\section{Conclusion}

The spectral moment method is robust in characterizing the energy spectra in the wavevector-frequency domain in two aspects. First, the lowest-order picture of the turbulence energy spectra is obtained in the wavevector-frequency domain by estimating the frequency shift velocity, the sweeping velocity, and the eigenvalues and eigenvectors of the second-order spectral moment tensor for the elliptic wavevector anisotropy. Second, the spectral moment method does not require the knowledge on the power-law index when estimating the sense of elliptical shape in the spectrum. The spectral index needs to be specified when one reproduces the wavevector spectrum using a model. The spectral moment method is advantageous in that the shift and sweeping velocities can be determined in a statistical way by repeating the analysis procedure at various wavenumbers. In contrast, the previously known method performs a fitting between the measurement and the spectrum model under a given set of free parameters (the shift velocity and the sweeping velocity) (Narita et al. 2013). In the spectral moment method, one can average the estimated shift and sweeping velocities over the wavenumbers.

The spectral moment method can be applied to various multi-point measurements in geophysical and space plasma measurements. The spectral moment method can also be used in the study of dispersion relation from the spectral measurements. The frequency estimate from the first-order moments can be compared with the theoretical branches of dispersion relation such a sound waves or Alfvén waves. The frequency estimate from the secondorder moments can be used as an error or an uncertainty measure of the dispersion relation study. The dispersion analysis in a mean flow, however, requires the knowledge of the flow velocity in order to correct for the Doppler shift.

\section{Author details \\ 1 Space Research Institute, Austrian Academy of Sciences, Schmiedlstraße 6, 8042 Graz, Austria. ${ }^{2}$ Institut für Geophysik und extraterrestrische Physik, Technische Universität Braunschweig, Mendelssohnstraße 3, 38106 Braunsch- weig, Germany. ${ }^{3}$ Institute of Physics, University of Graz, Universitätsplatz 5, 8010 Graz, Austria.}

\section{Acknowledgements}

This work is financially supported by the Austrian Space Applications Programme at the Austrian Research Promotion Agency, FFG ASAP-12, under contract 853994

\section{Competing interests}

The author declares that he has no competing interests.

\section{Publisher's Note}

Springer Nature remains neutral with regard to jurisdictional claims in published maps and institutional affiliations.

Received: 9 January 2017 Accepted: 16 May 2017

Published online: 26 May 2017
References

Balogh A, Carr CM, Acuña MH, Dunlop MW, BeekTJ, Brown P, Fornaçon KH, Georgescu E, Glassmeier K-H, Harris J, Musmann G, Oddy T, Schwingenschuh K (2001) The Cluster magnetic field investigation: overview of in-flight performance and initial results. Ann Geophys 19:1207-1217. doi:10.5194/angeo-19-1207-2001

Bruno R, Telloni D (2015) Spectral analysis of magnetic fluctuations at proton scales from fast to slow solar wind. Astrophys J Lett 811:L17. doi:10.1088/2041-8205/811/2/L17

Carbone V, Malara F, Veltri P (1995) A model for the three-dimensional magnetic field correlation spectra of low-frequency solar wind fluctuations during Alfvenic periods. J Geophys Res 100:1763-1778. doi:10.1029/94JA02500

Dasso S, Milano LJ, Matthaeus WH, Smith CW (2005) Anisotropy in fast and slow solar wind fluctuations. Astrophys J 635:L181-L184. doi:10.1086/499559

Escoubet CP, Fehringer M, Goldstein M (2001) The Cluster mission. Ann Geophys 19:1197-1200. doi:10.5194/angeo-19-1197-2001

Forman MA, Wicks RT, Horbury TS (2011) Detailed fit of "critical balance" theory to solar wind turbulence measurements. Astrophys J 733:76. doi:10.1088/0004-637X/733/2/76

Harding B, Tremblay C, Cousineau D (2014) Standard errors: a review and evaluation of standard error estimators using Monte Carlo simulations. Quant Methods Psychol 10:107-123. doi:10.20982/tqmp.10.2.p107

He G-W, Zhang J-B (2006) Elliptic model for space-time correlation in turbulent shear flows. Phys Rev E 73:055303R. doi:10.1103/PhysRevE.73.055303

He J, Tu C, Marsch E, Yao S (2012) Reproduction of the observed twocomponent magnetic helicity in solar wind turbulence by a superposition of parallel and oblique Alfvń waves. Astrophys J 749:86. doi:10.1088/0004-637X/749/1/86

Howes GG, Nielson KD (2013) Alfvén wave collisions, the fundamental building block of plasma turbulence. I. Asymptotic solution. Phys Plasmas 20:072302. doi:10.1063/1.4812805

Kraichnan RH (1964) Kolmogorov's hypotheses and Eulerian turbulence theory. Phys Fluids 7:1723-1734. doi:10.1063/1.2746572

Lehmann EL, Casella G (1998) Theory of point estimation. Springer, New York

Matthaeus WH, Goldstein ML, Roberts DA (1990) Evidence for the presence of quasi-two-dimensional nearly incompressible fluctuations in the solar wind. J Geophys Res 95:20673-20683. doi:10.1029/JA095iA12p20673

Narita Y, Glassmeier K-H, Motschmann U (2011) High-resolution wave number spectrum using multi-point measurements in space - the multi-point signal resonator (MSR) technique. Ann Geophys 29:351-360. doi:10.5194/ angeo-29-351-2011

Narita Y, Glassmeier K-H, Motschmann U, Wilczek M (2013) Doppler shift and broadening in solar wind turbulence. Earth Planets Space 65:e5-e8. doi:10.5047/eps.2012.12.002

Narita Y (2014) Four-dimensional energy spectrum for space-time structure of plasma turbulence. Nonlinear Process Geophys 21:41-47. doi:10.5194/ npg-21-41-2014

Narita Y (2015) Non-elliptic wavevector anisotropy for magnetohydrodynamic turbulence. Ann Geophys 33:1413-1419. doi:10.5194/ angeo-33-1413-2015

Narita Y (2017) Error estimate of Taylor's frozen-in flow hypothesis in the spectral domain. Ann Geophysics 35:325-331. doi:10.5194/ angeo-35-325-2017

Rème H, Aoustin C, Bosqued JM, Dandouras I, Lavraud B, Sauvaud JA, Barthe A, Bouyssou J, Camus Th, Coeur-Joly O, Cros A, Cuvilo J, Ducay F, Garbarowitz Y, Medale JL, Penou E, Perrier H, Romefort D, Rouzaud J, Vallat C, Alcaydè D, Jacquey C, Mazelle C, d'Uston C, Möbius E, Kistler LM, Crocker K, Granoff M, Mouikis C, Popecki M, Vosbury M, Klecker B, Hovestadt D, Kucharek H, Kuenneth E, Paschmann G, Scholer M, Sckopke N, Seidenschwang E, Carlson CW, Curtis DW, Ingraham C, Lin RP, McFadden JP, Parks GK, Phan T, Formisano V, Amata E, Bavassano-Cattaneo MB, Baldetti P, Bruno R, Chionchio G, Di Lellis A, Marcucci MF, Pallocchia G, Korth A, Daly PW, Graeve B, Rosenbauer H, Vasyliunas V, McCarthy M, Wilber M, Eliasson L, Lundin R, Olsen S, Shelley EG, Fuselier S, Ghielmetti AG, Lennartsson W, Escoubet CP, Balsiger H, Friedel R, Cao J-B, Kovrazhkin RA, Papamastorakis I, Pellat R, Scudder J, Sonnerup B (2001) First multispacecraft ion measurements in and near the Earth's magnetosphere with the identical Cluster ion spectrometry (CIS) experiment. Ann Geophys 19:1303-1354. doi:10.5194/angeo-19-1303-2001 
Saito S, Gary SP, Li H, Narita Y (2008) Whistler turbulence: particle-in-cell simulations. Phys Plasmas 15:102305. doi:10.1063/1.2997339

Shebalin JV, Matthaeus WH, Montgomery D (1983) Anisotropy in MHD turbulence due to a mean magnetic field. J Plasma Phys 29:525-547. doi:10.1017/S0022377800000933

Sorriso-Valvo L, Carbone V, Veltri P, Consolini G, Bruno R (1999) Intermittency in the solar wind turbulence through probability distribution functions of fluctuations. Geophys Res Lett 26:1801-1804. doi:10.1029/1999GL900270 Taylor GI (1938) The spectrum of turbulence. Proc R Soc Lond A 164:476-490. doi:10.1098/rspa.1938.0032
Wilczek M, Narita Y (2012) Wave-number frequency spectrum for turbulence from a random sweeping hypothesis with mean flow. Phys Rev $E$ 86:066308. doi:10.1103/PhysRevE.86.066308

Zhao X, He G-W (2009) Space-time correlations of fluctuating velocities in turbulent shear flows. Phys Rev E 79:046316. doi:10.1103/ PhysRevE.79.046316

\section{Submit your manuscript to a SpringerOpen ${ }^{\odot}$ journal and benefit from:}

- Convenient online submission

- Rigorous peer review

- Immediate publication on acceptance

- Open access: articles freely available online

- High visibility within the field

- Retaining the copyright to your article 